\title{
The leadership and followership
}

\section{challenges of doctors in training during}

\section{the COVID-19 pandemic}

\author{
Authors and affiliations \\ Dr Nathan Boardman (co-first author) \\ Senior House Officer \\ Unaffiliated
}

CF32 9GG

nboardman@doctors.org.uk

Jack Munro Berry (co-first author)

General Practice Registrar

Royal Cornwall Hospital

TR1 3L

UK

Jack.munro-berry@nhs.net

Professor Judy McKimm (corresponding author)

Emeritus Professor of Medical Education

Swansea University

SA2 8PP

UK j.mckimm@swansea.ac.uk 
All authors contributed equally to this paper.

\section{Conflict of interest}

The authors have no conflict of interest to report.

\section{Abstract}

Research carried out in 2016 by the authors investigated the challenges that doctors in training experience around leadership and followership in the UK National Health Service (NHS). The study explored contemporary healthcare leadership culture and the role of followership from the perspective of early career doctors. It found that the leadership and followership challenges for these doctors in training were associated with issues of social and professional identity, communication, the medical hierarchy, and relationships with senior colleagues (support and trust). These challenges were exacerbated by the busy and turbulent clinical environment in which they worked. To cope with various clinical situations and forms of leadership, doctors in training engage in a range of different followership behaviours and strategies.

The study raised implications for medical education and training and suggested that followership should be included as part of formal training in communication and team working skills. The importance of both leadership and followership in the delivery of safe and effective patient care has been brought sharply into focus by the COVID-19 pandemic. We revisit these challenges in light of the pandemic and its impact on the experiences of doctors in training. 


\section{Introduction}

The engagement of doctors and other health professionals in healthcare leadership and management is central for the delivery of high-quality clinical services (Spurgeon et al., 2011) and leadership development is now viewed as essential for every doctor. Traditional leadercentric teaching and research is insufficient to create and sustain efficient teamworking. Being able to work effectively in a team has been essential during the COVID-19 crisis when teams are put under increasing pressure to treat patients quickly, yet safely, whilst often working in new, temporary teams, and outside their usual scope of practice (Paixao et al., 2020). This involves being able to lead, manage and follow well (the leadership 'triad') (McKimm, O'Sullivan 2016), but doctors have not traditionally learned about followership, although this is changing (McKimm \& Vogan, 2020; Gordon et al 2015). During the pandemic, doctors at all levels are being asked to follow the leadership of medical directors, NHS chiefs and central governments on an unprecedented level. How and who doctors choose to follow is therefore critical to healthcare delivery.

\section{Background and context}

This article builds on the findings of a research study carried out in 2016 (McKimm et al., 2020; Jones et al. 2016) which analysed 51 Critical Incident Analyses (CIAs) and 51 analyses of workplace situations (WS) where poor or exemplary leadership was observed. These written pieces were produced by 51 doctors in training, from 5 different cohorts (2009-2014) on an academic programme in clinical leadership and management. Ethical approval for the study was granted by Leicester University. These doctors in training were well placed to deliver an informed and analytical account of contemporary leadership culture in the NHS and the 
leadership and followership challenges that they faced. The research identified four interconnected themes around leadership and followership:

1. Dealing with medical hierarchy

2. Their relationships with senior colleagues: Support and trust

3. Communicating with others

4. Developing professional identities

These challenges were exacerbated by the busy and turbulent clinical environment and the study found that, in order to cope with various clinical situations and forms of leadership, doctors in training engage in a range of different followership behaviours and strategies. We go on to discuss these challenges and themes in light of the COVID-19 pandemic.

\section{Followers and followership}

The study of followership (as distinct from leadership) is relatively recent but is an increasing focus of attention. Early work described 'typologies' of different types of follower, for example designating individuals to one of five typologies based upon their level of independent, critical thinking and level of activity towards a goal (Kelley 1988). Followers who are capable of independent critical thinking but are disengaged in achieving the team goal are described as 'alienated followers', who appear cynical and may see themselves as the rightful leader. 'Passive followers' or 'sheep' may appear engaged but, because of inexperience or an unwillingness to act independently, tend to follow the leader without question. 'Conformist followers' or 'yes people' look to their leader for direction, defer to their wishes and (like 'sheep') do not challenge. The exemplary, 'star' follower is actively 
engaged, acts on their own initiative (is a good deputy), and provides constructive, critical advice. Finally, the 'pragmatic followers' or 'fence-sitters' choose to behave in a way that best suits them at the time.

Our understanding of followership has subsequently developed to explore levels of engagement (Kellerman, 2008); the impact of followers on leaders' behaviour (Uhl-Bien et al., 2014); the role of followers in generating collective emotion, and followership and clinical teamworking (McKimm and Vogan 2020). Paradis suggests that followership is the ability to:

"recognise the shared purpose and interdependence of team members, 2) think independently and critically, and 3) show courage and a supportive team-oriented attitude when challenging leadership decisions or plans" (Paradis 2019).

Effective followers are therefore very similar to effective leaders: they manage themselves well; are committed to and fully engaged with the organisation and a purpose, principle, or person outside themselves; build their competence and focus their efforts for maximum impact; can influence appropriately, and are courageous, honest, and credible (Uhl-Bien et al., 2014; Gibbons et al., 2012; Bjugstad et al., 2006).

Followers who are independent, critical thinkers consider the impact of their actions rather than simply accepting the leader's instructions. To ensure patient safety and ownership of joint decisions, a culture is needed that facilitates team members to speak out and question those in leadership positions. Medical leaders therefore need to model good followership behaviours as well as demonstrate leadership, so that doctors in training learn how to follow well, rather than seeing this as a passive activity (Klingensmith, 2017). 
In this article, we explore followership, revisit the themes and challenges identified in the earlier research study in light of lessons learned during the 2020 COVID-19 pandemic, and discuss useful leadership and followership strategies.

\section{Dealing with Medical Hierarchy}

The medical profession has a recognisable and distinct hierarchy with consultants at the top, doctors in training (at various grades) in the middle and medical students at the bottom. Our earlier study found that this hierarchy can create a negative working environment where junior healthcare professionals feel a lack of confidence and are unwilling to challenge their superiors, even if they think their clinical judgement is wrong (McKimm et al., 2020). This workplace culture contributes to communication failures and significant reversible causes of patient harm (Sutcliffe et al., 2004). "If we are to improve patient safety then it is vital that students and doctors in training are enabled and equipped with the skills and confidence to challenge perceived authority when working in healthcare teams, while maintaining good working relationships" (Moneypenny et al., 2013).

In addition to developing the confidence and communication skills of doctors in training, senior clinicians must also aim to breakdown the negative effects of the medical hierarchy by promoting a culture of 'shared' or 'distributed' leadership (Paradis 2019). Here, "leadership is considered not to reside in one individual [i.e., the consultant]; it is an informal, social process where expertise is acknowledged to be distributed, boundaries to leadership are open and leadership emerges from within the connections of the organisation" (Swanwick, McKimm, 2011, p11). This approach creates a flattened hierarchy where constructive feedback is actively sought and colleagues are willing to have "difficult conversations" regardless of rank. This would promote the development of exemplary followers and help mitigate the risk of 
followers becoming alienated. The shift from 'red cape leadership', where the responsibility lies with one heroic leader, to 'shared leadership', is relevant to increasingly complex environments such as the NHS (especially in a crisis) where "one person cannot possibly know enough, see enough, understand enough to make perfect decisions all of the time" (Heffernan 2015). Clinical teams which hinge on the knowledge and expertise of one 'heroic' consultant will only ever be as smart as that individual and will struggle or fail when faced with issues outside their comfort zone (Ham et al., 2011).

Followers working in teams in which the traditional hierarchy is less strictly established feel more flexibility and confidence when discussing ideas with their superiors (Green et al., 2017). Healthcare teams that focus on the patient's needs and have clear goals and leadership stimulate creativity and innovation, and (perhaps more importantly from a patient safety perspective) allow discussion of potentially harmful practices (Broom, Broom, 2018; Ezziane et al., 2012; West, Sacramento, 2006). However, our study findings corroborated other research that indicates that most doctors in training still hold traditional understandings of leadership and followership, influenced by the hierarchical organisational structure and culture in which they work.(Gordon et al., 2015; Brennan, Davidson, 2019)

To encourage other team members to be effective followers in such hierarchies, traditional leaders may need to 'step back', allow the different skills of those around them to flourish, and develop a more flexible approach to the leader-follower relationship. This will help doctors in training to progress from subordinate to colleague and develop appropriate followership behaviours: being listened to, valued, and given more responsibility should help a former 'yes person' or 'sheep' develop into an 'exemplary follower'. 
Much more needs to be done to develop this culture of shared leadership, positive followership, and distributed responsibility. As one of the earlier study participants suggested: "as doctors we need to create a culture where everyone strives to ensure that patient safety is paramount, feels that they can identify weaknesses within practice and that individuals are not blamed" (P7)

\section{Relationships with senior colleagues}

Our study identified two sub-themes in this aspect: a lack of support for doctors in training from their senior colleagues, and the importance of mutual trust. Both these challenges were exacerbated by busy, turbulent clinical environments, and the pandemic has only increased these workplace pressures.

\section{Support}

A lack of support is another root cause of doctors in training's leadership failures due to understaffing, their own and seniors' work pressures and being "overwhelmed in terms of patient numbers." (P25). This leads to situations where they feel they have no-one to turn to and are therefore "... put under pressure to assume a leadership role when they might not feel competent" (P8). "We cannot change the human condition, but we can change the conditions under which humans work" (Reason 2000), therefore, whilst we cannot change the system pressures due to the pandemic, proactive measures need to be put in place to provide additional support, both 'after the fact' and 'in the moment'. Otherwise, doctors in training will not thrive as followers or as leaders and may default to less helpful followership behaviours, for example: over-conforming, automatically and unquestioningly carrying out 
their jobs, or becoming alienated, not working with the team, or over-challenging the leader.

In some situations, such as emergency care settings, or critical events involving basic and advanced life support, typical followership styles tend towards the 'yes person': this is not necessarily negative. The continual presence of more senior, experienced staff, and the need for quick and efficient responses to clear instructions and direction to ensure patient stability, requires 'obedience', however, despite more senior doctors taking the lead in most scenarios, individuals still needed to display 'star follower' characteristics to evaluate instructions and perform tasks appropriately, e.g.:

"Despite the obvious that the consultant was the team leader for this emergency scenario, everyone displayed their own individual leadership skills and worked well within the team...Flow of change in team leadership after arrival of consultant and registrar" (P29)

\section{Trust}

Followers need to trust their leaders, and vice versa. Trust between members and of their leaders is essential in team effectiveness, but when teams rapidly change their membership (such as in the pandemic), it can be hard to establish quickly (Kiffin-Petersen, 2003). As such, building relationships comprises, in part, gaining and maintaining the trust and support of colleagues (Mineo 2014). Followers are more willing to take on jobs when there is mutual respect between junior and senior and open conversations. They also display more qualities of the 'exemplary follower' if their leader is someone they have known for some time, has a 'good character', is clinically competent, and recognises when team members can be trusted to complete work in their own way. 
"Our initial trust in him was based on two principles: character and competence. If he had spent time second guessing his decisions, then we would have felt that the sincerity and honesty of his methods were flawed and a crisis of followership may ensue" (P4)

However, trust is not automatic. One foundation doctor described a situation in which their vigilance was not recognised by their senior, resulting in a poor outcome for their patient:

"I spent approximately 10 minutes bleeping various surgeons and members of my own team. Eventually I contacted my registrar who dismissively told me to contact the medical registrar on-call. I was awaiting a response... when one of the nurses put out the arrest call out for my patient" (P23)

In such cases, swift action should be taken to re-establish working leader-follower relationships. Top level leaders can frequently become detached from the reality of the dayto-day experience of staff and patients (Chaffer 2016) therefore actively enabling followers to develop their skills and roles and act innovatively may help develop 'transcendent' followers. These are followers that develop and display competence in terms of their management of relations with self (being self-aware and proactive in developing individual strengths), others (in relation to leaders and peers), and the organisation (collective maintenance and change), (Cunha et al., 2013).

\section{Communicating with others}

Many of the participants in our earlier study identified their own poor communication skills as a major underlying cause of their leadership failures. For example, one participant explained how they "communicated poorly with the nursing team about how sick [a] patient was, which caused some resistance in them carrying out jobs." (P13) In this scenario the 
patient quickly deteriorated and ultimately died. This was not necessarily avoidable as he was very unwell, and the participant's poor communication skills were certainly not the only factor that contributed to his suboptimal management. If the nurses were given clearer instructions, they may have better understood the urgency and reacted more appropriately. It is in these such scenarios where flexibility in followership styles can arise and may result in ultimately different outcomes. On the one hand, a 'yes person' may adhere to the instructions in a strict manner whether passively (unaware that those instructions may contribute to poor quality care) or to actively protect themselves from potential litigation or humiliation. However, it can also lead to the blossoming of transcendent followers, those able to critically analyse the instructions given and challenge the leaders' direction.

Being able to communicate effectively is an essential skill for a doctor, and poor communication between health professionals has been well established as an underlying cause of leadership failure and poor healthcare delivery (Donaldson et al 2014). Communication skills are also a significant component of leadership development that must be purposefully fostered in doctors in training: "the art of communication is the language of leadership" (Humes 2020). In Outcomes for Graduates (General Medical Council, 2018), the General Medical Council (GMC) has hugely strengthened the learning outcomes around leadership, teamworking and communication skills that UK medical graduates must be able to demonstrate. However, there is no mention of followership.

Handover is a key point where patient care can be compromised (Eggins, Slade, 2012) and the study identified how unprepared new doctors were e.g.:

"New doctors feel unprepared for handover and poor systems to facilitate handover are a barrier to ensuring effective continuity of care." (P19) 
Leadership and management development should be much more explicit in the undergraduate curriculum (Till et al., 2017) and, whilst an indicative undergraduate curriculum in medical leadership and management has recently been produced (Faculty of Medical Leadership \& Management (FMLM 2018), it is still early days for many medical schools, and research regarding the efficacy of leadership teaching methods at undergraduate level is still in its infancy. The FMLM guidance includes followership learning in the 'working within teams' section, suggesting that students should be able to 'demonstrate knowledge of a range of leadership and followership approaches and their appropriateness in various situations' (FMLM 2018, p13).

\section{Developing professional identities}

A 'social identity' is a person's knowledge that they belong to certain social groups (e.g., worker, parent) and such group membership has some emotional significance (Tajfel 1972). Professional identity relates to how an individual sees themselves (reflecting how others see them) as a professional. For doctors, this is established through early socialisation and enculturation, in the formative years as a medical student and as a doctor in training. Developing an appropriate professional identity is as important as developing medical knowledge and skills (Wilson et al., 2013). Without this, even if someone possesses all the technical competencies and attributes of a good doctor, they may lack the confidence and courage of their convictions to truly "be" a good doctor (Monrouxe, 2010).

Beliefs in the way doctors should function in the work environment, such as thinking they will operate with relative autonomy and hold authority over other healthcare professionals, is formed prior to medical school but develops throughout their careers (Horsburgh et al., 2006). Doctors in training (like everyone) have views on how 'good leaders' behave. 
"Implicit leadership theories help to explain some of the struggles faced by leaders who do not 'fit' into their followers' schemata, based not on leadership skills but on general attributes such as gender, profession, sexuality, disability, age, or race. In practical terms, this means that some leaders may have to work much harder to overcome deeply held (but not always articulated) beliefs about what leaders should look like, and how they should behave" (Mannion et al., 2015). As we have seen however, the doctor's own beliefs about themselves as a leader or follower may also influence (positively or negatively) their own perceptions of their impact on followers (McKimm et al., 2017). Such implicit leadership theories (ILTs) are very powerful and when these doctors in training feel they do not measure up to these high standards, then it can be damaging to their evolving (sometimes fragile) identities.

In medicine, the inbound trajectory into a community of practice involves an individual moving from student (a 'novice'), to a doctor in training and finally to an 'expert' consultant (Barrow et al., 2015; Farnsworth et al., 2016). Students and doctors in training are accorded 'legitimate peripheral participation' in the community of practice (Lave, Wenger, 2001) and such accorded legitimacy facilitates an individual's identity formation as they move from medical student, to doctor in training and beyond. An individual's desired professional identity therefore relies upon the acceptance of their identity claims by others, affirming that they are perceiving the individual's identity as legitimate (Brown, Toyoki, 2013; Sveningsson, Alvesson 2003). As such, this can result in multiple potentially contradictory narratives, ultimately resulting in an inability to communicate effectively to other group members and triggering an identity conflict, which presents challenges for leaders and 
followers alike (Croft et al., 2015). This can ultimately result in new graduates demonstrating followership patterns rooted in subordinacy, for example those of the yes person or alienated follower, ignoring problems or unwilling to question the leaders' decisions.

The earlier study found that a crisis in role and identity rendered many doctors in training inefficient in their clinical practice:

"Without really knowing what our role as a junior doctor consisted of, we spent the first few weeks... being heavily criticised by senior doctors and nurses for making drug errors, completing the wrong surgical forms for patients, delivering them to the wrong department, not completing pre-assessment clinic forms correctly, failing to take correct bloods from patients on admission and so on." (P59)

Prototypicality, defined as 'the extent to which followers perceive leaders to be representative of the group's identity', is particularly important in a healthcare environment where social and professional identities are profoundly linked (Tee et al., 2013). Doctors are also acutely aware of the scrutiny by the public of leaders who fail to behave in an exemplary manner, which risks not only alienating or scapegoating individuals but also threatens the salient identity of the entire group (Mannion et al., 2015). This is exacerbated currently, with further intense scrutiny on the health system as efforts are doubled to ensure hospitals are not overwhelmed by the wave of coronavirus cases and doctors being required to take leadership in specialities outside their own. 


\section{Conclusions}

Even in 'normal' times, doctors in training face many challenges, which are exacerbated during times of stress and transition. The challenges identified in our earlier research still have the potential to seriously impact on patient safety if they remain unaddressed. Arguably the most significant challenge which doctors in training face in leadership roles is the contemporary medical hierarchy which contributes to an unproductive and potentially toxic workplace culture. This is exacerbated by the poor communication skills of some doctors in training who are sometimes unable to deliver clear and concise instructions in leadership situations. Again, this has a significant effect on patient safety, particularly in handover processes. Finally, widespread understaffing and a turbulent clinical environment leaves doctors in training frequently unsupported, which often results in them assuming clinical leadership roles beyond their level of competence.

It is crucial that followers maintain the ability to challenge toxic or poor leadership and it is apparent that an underlying level of trust between team members facilitates effective followership. Effective collaboration and communication between leaders and followers are needed to achieve optimal patient outcomes. If doctors in training see themselves as passive (low in the medical hierarchy) or alienated (unsupported) types of followers, this will inhibit their ability to speak out and seek help when they need it.

Throughout the pandemic, doctors have been involved in scenarios with which they may be unfamiliar, and some individuals have had to work with teams and systems with which they are uncomfortable. It is in these situations that exemplary followers will thrive. Effective teamworking and followership are therefore critical, to promote collectivism and collaboration. Leaders also need to be great followers, willing to take the reins but pass 
them on when required (McKimm, Mannion, 2015). In the NHS, where a focus on relatively traditional leadership approaches dominates within a highly hierarchical establishment, nurturing effective followership as a core part of education and training in teamworking and communication skills is not only important, but potentially lifesaving.

\section{References}

Barrow M, McKimm J, Gasquoine S, Rowe D. Collaborating in healthcare delivery: exploring conceptual differences at the "bedside". Journal of Interprofessional Care. 2015 Mar 4;29(2):119-24.

Bjugstad K, Thach EC, Thompson KJ, Morris A. (2006) A fresh look at followership: A model for matching followership and leadership styles. Journal of Behavioural and Applied Management. 2006;7:304-19.

Brennan PA, Davidson M. Improving patient safety: we need to reduce hierarchy and empower junior doctors to speak up. BMJ. 2019 Jul 2;366:14461.

Broom J, Broom A. Fear and hierarchy: critical influences on antibiotic decision-making in the operating theatre. Journal of Hospital Infection. 2018 Jun 1;99(2):124-6.

Brown AD, Toyoki S. Identity Work and Legitimacy. Organization Studies. 2013;34(7):875-96.

Chaffer D. Effective Leadership: A Cure for the NHS? Boca Raton, FL: CRC Press; 2016.

Croft C, Currie G, Lockett A. The Impact of Emotionally Important Social Identities on the Construction of a Managerial Leader Identity: A Challenge for Nurses in the English National Health Service. Organization Studies. 2015;36(1):113-31. 
Cunha MPE, Rego A, Clegg S, Neves P. The case for transcendent followership. Leadership. 2013;9(1):87-106.

doi: https://doi.org/10.1136/bmj.1348 https://www.bmj.com/content/364/bmj.1348/rr-2

Donaldson LJ, Panesar SS, Darzi A. Patient safety-related hospital deaths in England: Thematic analysis of incidents reported to a national database, 2010-2012. PLoS Medicine.

2014;11(6):e1001667

Eggins S, Slade D. Clinical handover as an interactive event: informational and intercactional communication strategies in effective shift-change handovers. Communication \& medicine. 2012;9(3):215-27.

Ezziane Z, Maruthappu M, Gawn L, Thompson EA, Athanasiou T, Warren OJ. Building effective clinical teams in healthcare. Journal of health organization and management. 2012 Aug 3.

Faculty of Medical Leadership and Management (2018) Medical leadership and management: an indicative undergraduate curriculum available at https://www.fmlm.ac.uk (accessed 26 November 2020)

Farnsworth V, Kleanthous I, Wenger-Trayner E. Communities of practice as a social theory of learning: A conversation with Etienne Wenger. British Journal of Educational Studies. 2016 Apr 2;64(2):139-60.

General Medical Council. Outcomes for Graduates 2018. 2018. Available from: https://www.gmcuk.org/-/media/documents/dc11326-outcomes-for-graduates-2018_pdf-75040796.pdf [Accessed 6 November 2020]

Gibbons A, Bryant D. Followership: the forgotten part of doctors' leadership BMJ 2012; 345 :e6715

Gordon L, Rees CE, Ker JS, Cleland J. Leadership and followership in the healthcare workplace: exploring medical trainees' experiences through narrative inquiry. BMJ Open. 2015;5(12):11. 
Green B, Oeppen RS, Smith DW, Brennan PA. Challenging hierarchy in healthcare teams-ways to flatten gradients to improve teamwork and patient care. British Journal of Oral and Maxillofacial Surgery. 2017 Jun 1;55(5):449-53.

Ham C, Baker GR, Docherty J, Hockey P, Lobley K, Tugendhat L, Walshe K. The future of leadership and management in the NHS: no more heroes. Report from the King's Fund on Leadership and Management in the NHS. 2011.

Heffernan, M. the Future of Leadership [Radio broadcast]. BBC Radio 4. 31 ${ }^{\text {st }}$ December 2015 20:00. Available from: http://www.bbc.co.uk/programmes/b06shyrh

Horsburgh M, Perkins R, Coyle B, Degeling P. The professional subcultures of students entering medicine, nursing and pharmacy programmes. J Interprof Care. 2006;20(4):425-31.

Humes, J. The art of communication is the language of leadership. Available from:

http://www.freshbusinessthinking.com/the-art-of-communication-is-the-language-of-leadership/. [Accessed 21 November 2020]

Jones PK, Munro-Berry J. McKimm J. Junior doctors as leaders: "We struggle to communicate, feel unsupported and lack confidence". Oral presentation at AMEE 2016, Barcelona, Spain, Aug 27-31

Kellerman B. How followers are creating change and changing leaders. Boston, MA: Harvard School Press; 2008.

Kelley RE. In praise of followers. Harvard Business Review. 1988;66(6):142-8.

Kiffin-Petersen S. Trust in leadership: An examination of its role in the effectiveness of work teams. Australian Journal of Psychology. 2003;55:133-.

Klingensmith ME. Leadership and followership in surgical education. American Journal of Surgery. 2017;213(2):207-11. 
Lave J, Wenger E. Legitimate peripheral participation in communities of practice. In Supporting lifelong learning 2001 Nov 22 (pp. 121-136). Routledge.

Mannion H, McKimm J, O'Sullivan H. Followership, clinical leadership and social identity. British Journal of Hospital Medicine. 2015;76(5):270-4.

McKimm J, Boardman N, Vogan C, Munro-Berry J. Followership: Challenges for Leadership Improvement: Poster presented at Leaders in Healthcare Conference 2020. $5^{\text {th }}$ Virtual Annual Conference produced by the Faculty of Medical Leadership and Management; 2020, Nov 17-20

McKimm J, Mannion H. Medical leadership-we need more good followers. The Lancet. 2015 Oct $17 ; 386(10003): 1532$.

McKimm J, O'Sullivan H. When I say... leadership. Medical education. 2016 Aug 26;9(50):896-7. McKimm J, Vogan C, Mannion H. Implicit leadership theories and followership informs understanding of doctors' professional identity formation: A new model. The Asia Pacific Scholar. 2017;2(2):18-23.

McKimm J, Vogan CL. Followership: much more than simply following the leader. BMJ Leader. 2020; 4: $41-44$

Mineo DL. The Importance of Trust in Leadership. Research Management Review. 2014;20(1):n1. Moneypenny MJ, Guha A, Mercer SJ, O'Sullivan H, McKimm J. Don't follow your leader: challenging erroneous decisions. British journal of hospital medicine. 2013;74(12):687-90.

Monrouxe, L. (2010). Identity, identification and medical education: why should we care? Medical Education, 44(1), pp.40-49.

Paixão G, Mills C, McKimm J, Hassanien MA, Al-Hayani AA. Leadership in a crisis: doing things differently, doing different things. British Journal of Hospital Medicine. 2020 Nov 17:1-9. 
Paradis (2019) Rapid response to Educating doctors as leaders and followers, BMJ 2019; 364:1348

Reason J. Human error: models and management. BMJ : British Medical Journal. 2000;320(7237:768-

70.

Spurgeon P, Clrak J, Ham C, Keogh B, Medical Leadership: From the dark side to centre stage. London: Radcliffe Publishing; 2011

Sutcliffe KM, Lewton E, Rosenthal MM. Communication failure: an insidious contributor to medical mishaps. Academic medicine: Journal of the Association of American Medical Colleges. 2004;79(2):186-94

Sveningsson S, Alvesson M. Managing managerial identities: Organizational fragmentation, discourse and identity struggle. Human Relations. 2003;56(10):1163-93.

Swanwick T \& McKimm J. ABC of Clinical Leadership. Blackwell Publishing. 2011. p.11.

Tajfel H. Social categorization. English manuscript of 'La categorisation sociale. Introduction a la Psychologie Sociale. 1972;1:272-302.

Tee EYJ, Paulsen N, Ashkanasy NM. Revisiting followership through a social identity perspective: The role of collective follower emotion and action. Leadership Quarterly. 2013;24(6):902-18.

Till A, McKimm J, Swanwick T (2017) Twelve tips for integrating leadership development into undergraduate medical education. Medical Teacher, Oct 26:1-7. Doi:

10.1080/0142159X.2017.1392009

Uhl-Bien M, Riggio RE, Lowe KB, Carsten MK. Followership theory: A review and research agenda. Leadership Quarterly. 2014;25(1):83-104. 
West MA, Sacramento CA. Flourishing in teams: Developing creativity and innovation. Creative management and development. 2006; 3:25-44.

Wilson, I., Cowin, L., Johnson, M. and Young, H. (2013). Professional Identity in Medical Students:

Pedagogical Challenges to Medical Education. Teaching and Learning in Medicine, 25(4), pp.369-373.

\section{Keywords}

Leadership; Followership; Covid-19; Training; Education 